\title{
Silver Nanowire Electrodes: Conductivity Improvement Without Post-treatment and Application in Capacitive Pressure Sensors
}

\author{
Jun Wang $\cdot$ Jinting Jiu $\cdot$ Teppei Araki $\cdot$ Masaya Nogi $\cdot$ Tohru Sugahara $\cdot$ Shijo Nagao $\cdot$ Hirotaka Koga $\cdot$ \\ Peng He $\cdot$ Katsuaki Suganuma
}

Received: 22 August 2014/Revised: 16 October 2014/ Accepted: 20 October 2014/Published online: 14 November 2014

(C) The Author(s) 2014. This article is published with open access at Springerlink.com

\begin{abstract}
Transparent electrode based on silver nanowires (AgNWs) emerges as an outstanding alternative of indium tin oxide film especially for flexible electronics. However, the conductivity of AgNWs transparent electrode is still dramatically limited by the contact resistance between nanowires at high transmittance. Polyvinylpyrrolidone (PVP) layer adsorbed on the nanowire surface acts as an electrically insulating barrier at wire-wire junctions, and some devastating post-treatment methods are proposed to reduce or eliminate PVP layer, which usually limit the application of the substrates susceptible to heat or pressure and burden the fabrication with high-cost, time-consuming, or inefficient processes. In this work, a simple and rapid pre-treatment washing method was proposed to reduce the thickness of PVP layer from 13.19 to $0.96 \mathrm{~nm}$ and improve the contact between wires. AgNW electrodes with sheet resistances of 15.6 and $204 \Omega \mathrm{sq}^{-1}$ have been achieved at transmittances of 90 and $97.5 \%$, respectively. This method avoided any post-treatments and popularized the application of high-performance AgNW transparent electrode on more substrates. The improved AgNWs were successfully employed in a capacitive pressure sensor with high transparency, sensitivity, and reproducibility.
\end{abstract}

Keywords Silver nanowire $\cdot$ Pre-treatment $\cdot$ Transparent electrode $\cdot$ Pressure sensor

\section{Introduction}

Transparent electrodes are regarded as essential components in optoelectronic applications such as solar cells, touch screens, organic light-emitting diodes, and sensor devices [1-4], and indium tin oxide (ITO) thin films are the

Electronic supplementary material The online version of this article (doi:10.1007/s40820-014-0018-0) contains supplementary material, which is available to authorized users.

J. Wang · P. He $(\bowtie)$

State Key Laboratory of Advanced Welding and Joining, Harbin Institute of Technology, Harbin 150001, People's Republic of China

e-mail: hithepeng@hit.edu.cn

J. Wang · J. Jiu ( $₫)$. T. Araki · M. Nogi · T. Sugahara ·

S. Nagao $\cdot$ H. Koga $\cdot$ K. Suganuma

The Institute of Scientific and Industrial Research, Osaka

University, Osaka, Ibaraki 567-0047, Japan

e-mail: jiu@eco.sanken.osaka-u.ac.jp most widely used material for such applications. However, there are several drawbacks to use ITO thin film, such as the inherent brittleness, the expensive deposition process, and also the emerging indium scarcity. Several alternatives have been investigated, including carbon nanotubes (CNTs), graphene, conductive polymer, and metal nanowires [5-7]. Among these, transparent electrode based on silver nanowire (AgNW) networks is being studied intensively and attracting commercial interest owing to their great potential for flexible, cost-efficient, and large-scale fabrication [8-11].

Although bulk silver exhibits very low electrical resistivity, the conductivity of AgNW networks is limited, especially at high transmittance, by the contact resistance between wires due to the residual of polyvinylpyrrolidone (PVP) layer, which is usually employed as the capping agent to control nanostructure size and disperse nanowires during AgNW synthesis [12,13]. Several methods have been developed to enhance the contact between nanowires, such as high-temperature (above $200{ }^{\circ} \mathrm{C}$ ) or long-duration 
thermal annealing $[14,15]$, rinsing and pressure treatment [16], and photonic sintering [17]. Other techniques such as nanosoldering, microwire enhancement, and galvanic displacement aim to remove the PVP layer or enlarge the contact area at wire-wire junctions [3, 18, 19]. However, all of these are the post-treatment methods after AgNWs are deposited on the substrate or the surface of device. Therefore, not only do these methods complicate the fabrication process, they also inevitably influence the performance of the heat-sensitive, pressure-sensitive, or chemical-sensitive substrates.

Recently, a new strategy was proposed using long nanowires to reduce the number of wire-wire junctions in conductive paths, thereby leading to low sheet resistance [20,21]. However, the PVP-induced resistance problem still has not been solved. The residual PVP layer adsorbing on the surface of AgNW still acts as an electrically insulating barrier at the wire-wire junctions and undermines the conductivity of the electrode. As the saying goes, "sharpen the knife before cutting the wood," i.e., high-performance AgNW ink is prerequisite before the fabrication of AgNW transparent electrode. Hence, decreasing the thickness of PVP layer beforehand will be a suitable, simple, and effective method for conductivity improvement. Unfortunately, many efforts are still focused on various post-treatments of AgNW electrodes as mentioned above. So far, few reports have paid attention to the important process to improve AgNW ink before electrode fabrication. AgNW paste washed by water has been reported to joint copper at room temperature without pressure [22], but this close-packed AgNW layer with thickness over $20 \mu \mathrm{m}$ was quite different from transparent electrode. The actual effect of filtration washing and sonication dispersing process could improve the conductivity of AgNW films [15]; however, it is too time-consuming to produce nanoscale ink by filtration in practical applications. Moreover, the washing effect on long AgNW has not been discussed. In this paper, a simple and rapid washing method is proposed to tailor the thickness of PVP layer on the surface of very long AgNWs, and the nanowire ink for high-performance transparent electrode regardless of substrate properties was achieved accordingly. Solvents and washing parameters were carefully selected and adjusted to meet the dispersion and preservation requirements of the nanowires. Finally, as-washed AgNWs were used in capacitive pressure sensor, which showed high transparency, sensitivity, and reproducibility.

\section{Experimental}

\subsection{Preparation of AgNWs}

AgNWs for the preparation of transparent electrodes were synthesized using a one-step polyol method starting with two solutions. For the first, $1.0 \mathrm{~g}$ silver nitrate was dissolved in $40 \mathrm{~mL}$ ethylene glycol at room temperature, while for the second, $0.8 \mathrm{~g}$ PVP $(\mathrm{Mw}=360,000)$ was gradually dissolved in $50 \mathrm{~mL}$ ethylene glycol at $60{ }^{\circ} \mathrm{C}$ while stirring at $300 \mathrm{rpm}$. After complete dissolution, the two solutions were mixed and $13.6 \mathrm{~g}$ of $\mathrm{FeCl}_{3}$ solution $\left(600 \mu \mathrm{mol} \mathrm{L}^{-1}\right.$ in ethylene glycol) was added to the mixture at room temperature and stirred at $300 \mathrm{rpm}$ for $3 \mathrm{~min}$. The mixture was then heated at $110{ }^{\circ} \mathrm{C}$ without stirring for a 12 -h redox reaction. Finally, the solution was mixed with acetone at a volume ratio of 1:4 to precipitate AgNWs for the subsequent steps.

\subsection{Washing Method}

Thickness tailoring of PVP nanolayer was performed by first mixing AgNW precipitate with ethanol at a mass ratio of $1: 15$ and stirring at $150 \mathrm{rpm}$ for $15 \mathrm{~min}$ at room temperature, followed by centrifugation of the suspension at 3,000 rpm for $3 \mathrm{~min}$. The supernatant was carefully decanted, and the residual precipitate was dispersed in ethanol and prepared for further washing. This procedure constitutes one cycle of ethanol washing (E1) and was applied twice, thrice, or four times to obtain three more AgNW inklabeled E2, E3, and E4. Then, E4-AgNWs were mixed with deionized (DI) water at either 25 or $90{ }^{\circ} \mathrm{C}$, with stirring at $150 \mathrm{rpm}$ for $15 \mathrm{~min}$, to obtain W25-AgNWs and W90AgNWs, respectively. Meanwhile, E4-AgNWs were also mixed with dimethylformide (DMF), at either 25 or $140{ }^{\circ} \mathrm{C}$, with stirring at $150 \mathrm{rpm}$ for $15 \mathrm{~min}$ to form D25AgNWs and D140-AgNWs, respectively. The ink in DI water and DMF were then centrifuged at $3,000 \mathrm{rpm}$ for 3 min to obtain AgNW precipitate. Since the wetting properties of these three solvents on PET were different, the AgNWs were finally dispersed in ethanol for coating. All these AgNW inks were fixed at $1.2 \mathrm{wt} \%$ concentration. All the reagents mentioned above were purchased from Wako Pure Chemical Industries, Ltd.

\subsection{Fabrication of AgNW Transparent Electrodes}

PET films with a thickness of $100 \mu \mathrm{m}$ were employed as substrates for AgNW electrodes. The PET substrates were cleaned in ethanol with ultrasonic treatment and then dried in air. The well-dispersed AgNW inks were drop-coated on the PET substrates for various transmittances at $550 \mathrm{~nm}$ wavelength. The dropped ink spread on the surface until uniformly coating the substrate, and the specimens were dried in air for 2-3 min until the solvent is evaporated. AgNW ink was also coated on glass beaker, PET bottle, tissue paper, and bacterial cellulose to verify its adaptability on various substrates. Coating method for glass beaker and PET bottle was dip-coating, and for tissue paper and bacterial cellulose was also drop-coating. 


\subsection{Fabrication of Pressure Sensors}

AgNW transparent electrodes on PET substrates were employed in capacitive pressure sensors. Pure PVP was dissolved in ethanol at $5 \mathrm{wt} \%$ concentration. The PVP solution was spin-coated on the as-prepared AgNW electrodes at room temperature. After drying in air for about $30 \mathrm{~s}$, two pieces of sandwiched PET/AgNWs/PVP structures were assembled with the two PVP layers in contact. Two pieces of conductive tapes were pasted on AgNW electrodes for the capacitance measurement.

\subsection{Measurements and Characterization}

The morphology of the PVP layer adsorbed on the AgNWs was investigated by optical microscope (VHX-600, Keyence), scanning electron microscope (SEM, SU8020, Hitachi High-Technologies), and transmission electron microscope (TEM, JEM-2100, JEOL). The sheet resistance of $30 \mathrm{~mm} \times 20 \mathrm{~mm}$ AgNW electrodes was measured using a four-point probe meter (Loresta GP T610, Mitsubishi Chemical Analytech). The transmittance investigated here was the transmittance of parallel light and does not include the transmittance of diffused light. The parallel transmittance $\left(T_{\mathrm{p}}\right)$ of the AgNW electrode for wavelengths in the range $300-900 \mathrm{~nm}$ was measured using a UV-visible/near-infrared spectrophotometer (V670, JASCO). The testing window size of the spectrophotometer is $12 \mathrm{~mm} \times 6 \mathrm{~mm}$, and three different places of one sample were tested and the average value was used as the transmittance for each sample. Thermogravimetry analysis (TGA) was carried out on a thermal analyzer (TG-DTA 2000SA, Netzsch Japan). The electrode haze was measured using a D65 illumination haze meter with a strong visible light source (HZ-V3, Suga Test Instruments). For the capacitive sensor, changes in capacitance were measured using a digital multimeter (34410A, Agilent Technologies).

\section{Results and Discussion}

Transparent electrodes are designed to achieve both outstanding electrical conductivity and optical transmittance; however, these two properties are seemingly negatively correlated, since the higher density of nanowires leads to the increase of conductivity and also the decrease of transparency. This dilemma could be resolved by employing long AgNWs. For the one-step wet chemistry method, the length and diameter of $\mathrm{AgNWs}$ varied drastically with the reaction temperature and the stirring speed [12]. In this study, the procedure for growing AgNWs was modified as at low temperature of $110{ }^{\circ} \mathrm{C}$ without stirring. The as-synthesized AgNWs, shown in Fig. 1, had an average length of $89.5 \mu \mathrm{m}$ and an average diameter of $84.2 \mathrm{~nm}$. During the synthesis and growth of AgNWs, the PVP capping agent was gradually adsorbed on the surface of the newly emerging silver crystals and grew with the crystals to be a nanolayer on the surface of the nanowire, formed from the merging of the crystals (Fig. 1c inset). The thickness of the nanolayer ranged from 5.96 to $20.13 \mathrm{~nm}$ with an average of $13.19 \mathrm{~nm}$ (Fig. 1c). The PVP layer prevents the agglomeration of AgNWs due to steric repulsion [23], while too thick layer also increases the contact resistance. Therefore, the thickness of PVP layer needs to be tailored appropriately.

Many post-processing methods, such as heating and pressing, have been proposed to thin down the PVP layer. In fact, it is known that PVP dissolves well in various solvents such as water, methanol, ethanol, acetic acid, chloroform, and so on [24]. Therefore, it is feasible to use these solvents to reduce the thickness of PVP layer. In this study, a simple stirring, washing, and centrifugation method was employed, and its effect on the thickness of the PVP layer was investigated. Figure 2 shows that the average thickness is dramatically reduced to $5.74 \mathrm{~nm}$ after a single washing cycle using ethanol and then decreased gradually down to $2.43 \mathrm{~nm}$ after four cycles. The TEM images shown in the inset of Fig. 2 highlight the thickness change of PVP layer on the surface of AgNWs during washing. The thickness visibly decreased compared with that of the original AgNWs and gradually thinned after each washing cycle. This trend is also verified by the thermogravimetric analysis (TGA) shown in Fig. S1. For the untreated AgNWs samples, the weight loss indicates intense evaporation of organic residues. The rapid weight loss occurring between $\sim 300$ and $450{ }^{\circ} \mathrm{C}$ was attributed to the evaporation and decomposition of residual byproducts and PVP [22]. For E4-AgNWs, the weight loss at high temperature is much less, demonstrating that after four cycles of washing, a mass of PVP and byproducts had been removed.

The AgNWs washed in ethanol were used to fabricate electrodes on PET substrates at room temperature. Figure 3 shows the relationship between the electrical performance and the optical transmittance of the electrodes. The deviations of measured sheet resistance values were usually less than $\pm 1 \%$ of the average value at each measuring point. When the AgNW loading decreased, the transmittance increased but so did the sheet resistance. However, washing drastically decreased the resistance while maintaining the transmittance. For example, the resistances from zero to four washing cycles were $195,50.8,32.0,19.5$, and $15.6 \Omega \mathrm{sq}^{-1}$, respectively, with the same transmittance of ca. $90 \%$. The results indicate that washing gradually removed the PVP layer leading to lower contact resistance between AgNWs. Importantly, the reductions in resistance were more notable at higher transmittance. For instance, at $85 \%$ transmittance, the resistance dropped from 30 to $10 \Omega \mathrm{sq}^{-1}$ after four 
Fig. 1 AgNWs were successfully synthesized by one-step polyol method using PVP as a capping agent. a,b The length and diameter distribution of the as-synthesized AgNWs. Inset: morphology of AgNWs, low resolution (Scale $b a r=50 \mu \mathrm{m})$ and high resolution (Scale bar $=1 \mu \mathrm{m})$. c The thickness distribution of PVP nanolayer on the surface of AgNWs. Inset: the TEM image of individual AgNW with PVP nanolayer. Scale bar $=20 \mathrm{~nm}$

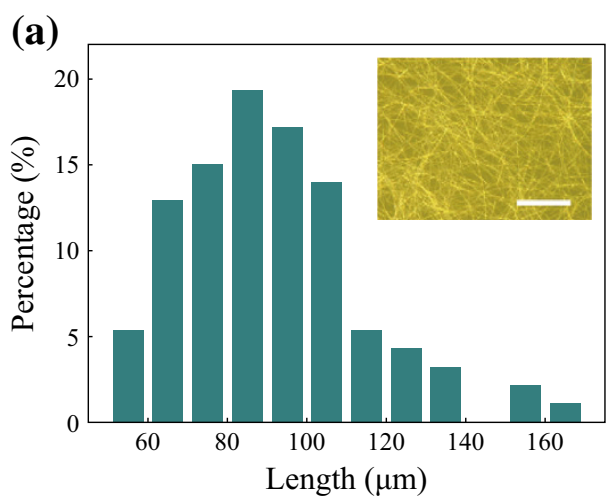

(b)

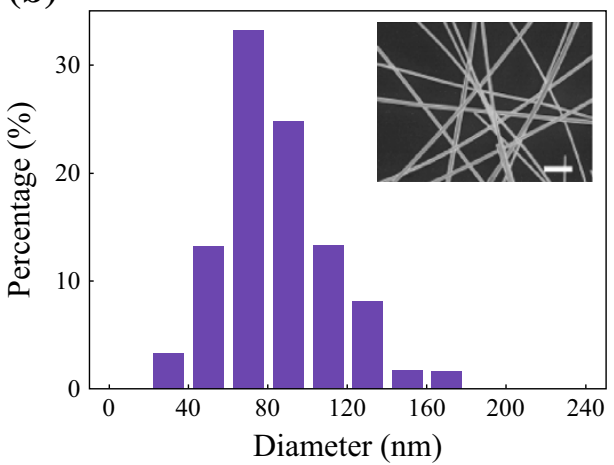

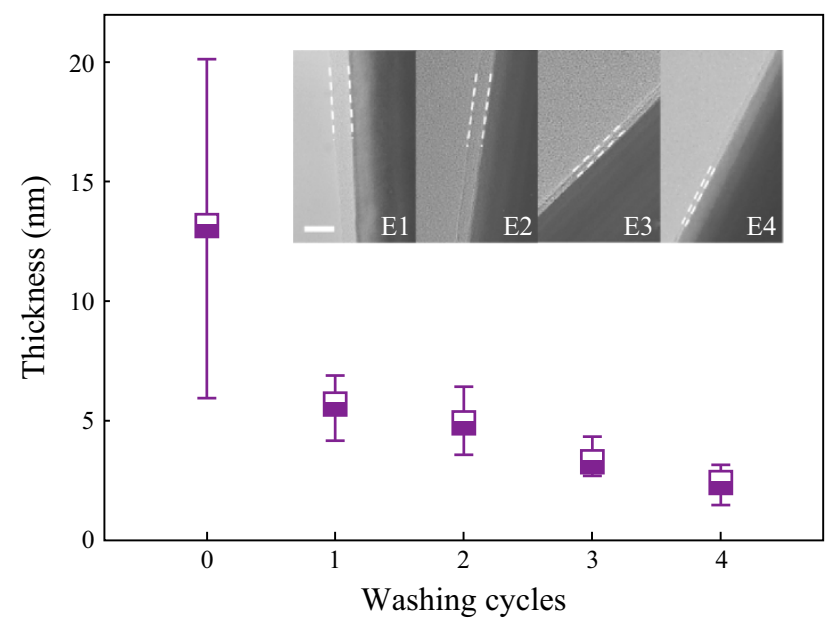

Fig. 2 Average thickness distribution of PVP nanolayer. Inset: TEM images of AgNWs washed with ethanol for different cycles. Scale bar $=10 \mathrm{~nm}$

cycles of washing, while at $95 \%$ transmittance, the drop is more dramatic, from $5 \times 10^{4}$ to $93.0 \Omega \mathrm{sq}^{-1}$. At low transmittance, dense AgNW percolation networks leaded to a large number of conductive paths, and this balanced the deleterious effect of high contact resistance at the wire-wire junctions (see Fig. S2). At high transmittance, however, there were fewer conductive paths, so the effect of contact resistance was more pronounced. Indeed, according to the percolation theory of conductive networks [25], sheet resistance would dramatically increase at the critical concentration of $\mathrm{AgNWs}$, i.e., percolation threshold, as the transmittance increased. In the present work, a thin PVP nanolayer made it possible to reduce percolation threshold and achieve low sheet resistance with a limited number of conductive paths. Moreover, within the measurement range, no clear correlation was observed between the thickness of the PVP nanolayer and the transmittance or haze of the fabricated electrode (see Fig. S3). This could be due to the thinness of the PVP nanolayer compared with AgNW and the high transmittance of PVP thin layer itself. Tailoring the thickness of the PVP nanolayer markedly improved the electrical characteristics of the electrodes with little or no effect on their optical properties. Summarily, it is necessary to wash AgNWs for four cycles using ethanol to reduce the PVP layer thickness to about $2.5 \mathrm{~nm}$ and achieve sheet resistance below 10 and $100 \Omega \mathrm{sq}^{-1}$ for transmittance below 82 and $95 \%$, respectively. However, since the reduction is lower after each cycle, the gains from washing more than four times were expected to be minor.

In order to further improve the contact between wires to achieve high conductivity, other solvents like DI water and dimethylformamide (DMF), which are better solvents for dissolving PVP (see Fig. S4), were used to wash the treated AgNWs. After washing with ethanol, E4-AgNWs were further 


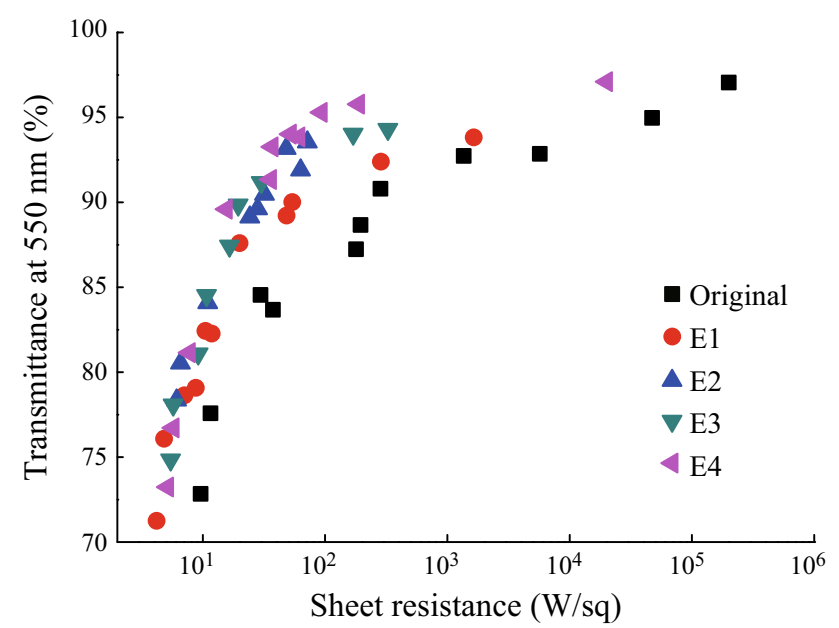

Fig. 3 Sheet resistance of AgNW electrodes with transmittances at $550 \mathrm{~nm}$ wavelength. Electrodes with original AgNWs were applied to compare with the ones with AgNWs washed in ethanol for different cycles

treated in DMF or water, and the evolution of PVP nanolayer morphology was investigated as shown in Fig. 4. The average thickness decreased from $2.43 \mathrm{~nm}$ to approximately $1 \mathrm{~nm}$. Washing at higher temperature $\left(140{ }^{\circ} \mathrm{C}\right.$ for DMF and $90{ }^{\circ} \mathrm{C}$ for DI water) led to greater reductions in thickness due to easier dissolution of PVP at higher temperature.

The sheet resistances of electrodes employing these four kinds of AgNWs were also compared with the ones employing E4-AgNWs (Fig. 4b). A similar pattern emerged that high-transmittance electrodes were more sensitive to changes in the thickness of the PVP nanolayer: for transmittance below $95 \%$ at the wavelength of $550 \mathrm{~nm}$, the sheet resistance was similar to that of ethanol-treated samples, while for electrodes with higher transmittance, a clear reduction in the sheet resistance was obtained. For example, the sheet resistance of electrodes using W90AgNWs dramatically dropped from $2.1 \times 10^{6}$ to $204 \Omega \mathrm{sq}^{-1}$ at the transmittance of $97.5 \%$. To the best of our knowledge, this result is the lowest value ever reported without any post-treatment and far exceeds that of ITO.

It should be noticed that the agglomeration of nanowires gradually emerged and finally precipitated when washing time in DI water or DMF was prolonged, corresponding to the excessive removal of PVP. The agglomeration degraded the dispersion of nanowires and thus the electrode performance at high transmittance. Therefore, it is important to maintain well-distributed nanowire percolation networks while reducing the contact resistance of individual wire-wire junction. In other words, the washing parameters should be carefully controlled to obtain PVP layer with a thickness optimal for peak electrical performance. The washing parameters included washing
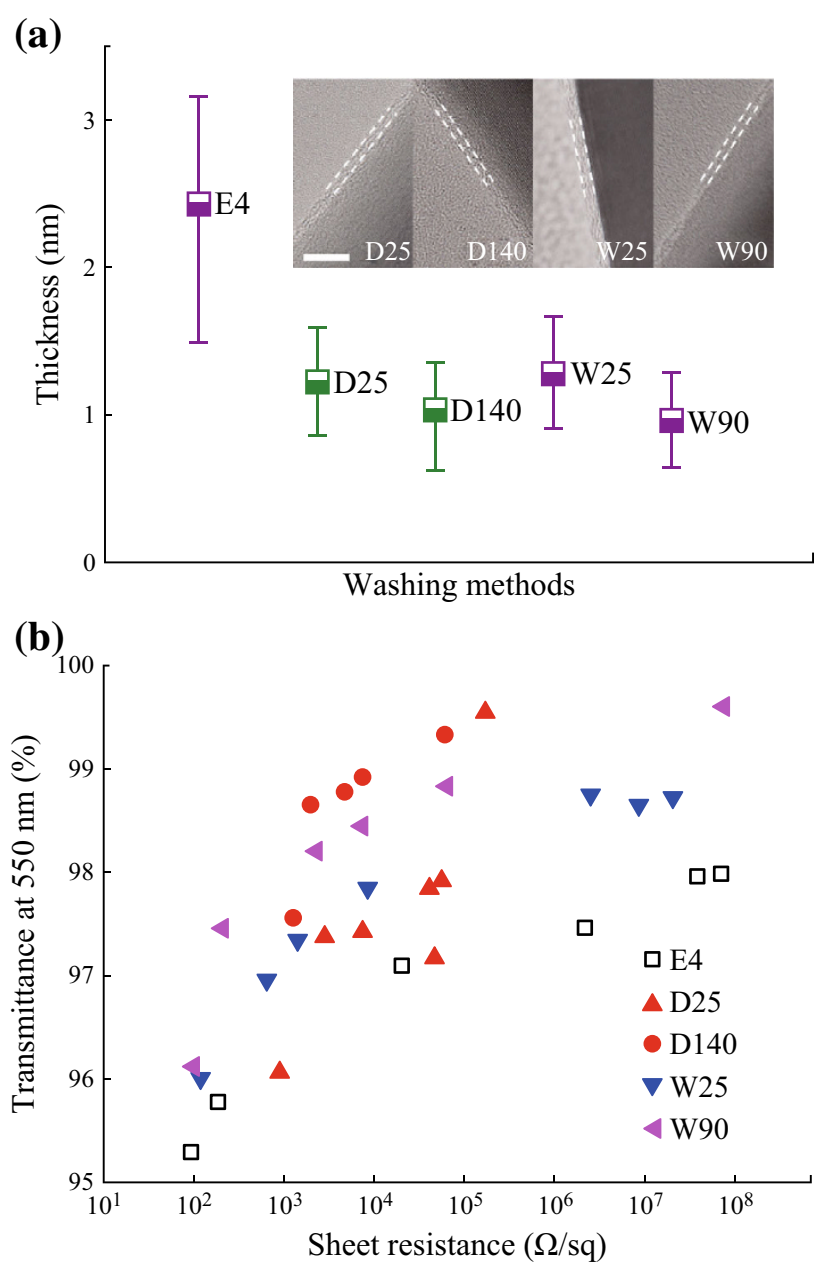

Fig. 4 a Average thickness of tailored PVP nanolayer before and after further washing treatment in DMF or DI water. Inset: morphology of tailored PVP nanolayer after further dissolution treatment. Scale bar $=5 \mathrm{~nm}$. b Sheet resistance of AgNW electrodes with high transmittance above $95 \%$ at $550 \mathrm{~nm}$ wavelength, employing $\mathrm{E} 4-\mathrm{AgNWs}$ and $\mathrm{AgNWs}$ further treated in DMF or DI water

temperature, washing times, stirring speed, and the solvent type. Simple, time-saving or cost-effective washing pretreatment could be easily achieved with different washing parameters to improve the electrical performance for various applications of AgNW transparent electrodes.

Figure 5 shows the comparison of the optoelectrical performances of the W90-AgNWs electrodes with those from previously published studies. It should be noted that our electrodes were fabricated at room temperature without any post-treatment. Electrodes produced by this simple washing method had much lower sheet resistance compared with long AgNWs [21] and showed similar or even lower values compared with AgNWs with an average length of $95.1 \mu \mathrm{m}$ annealed at $250{ }^{\circ} \mathrm{C}$ for $2 \mathrm{~h}$ [26]. Clearly, the electrodes also performed better than electrodes with short annealed AgNWs [26] and better than 


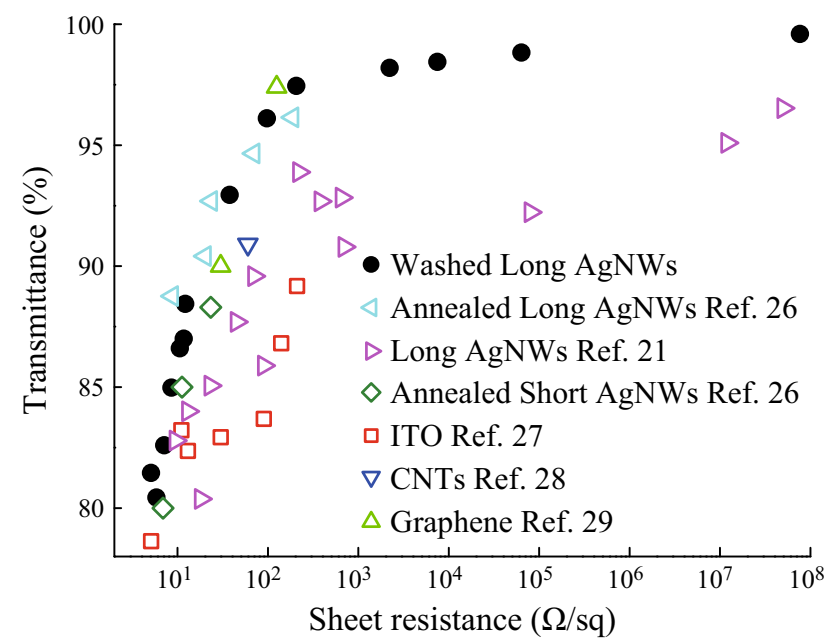

Fig. 5 The optoelectrical performance of electrodes with W90AgNWs compared with that of the other reported transparent electrodes

other conductive materials such as ITO [27], CNTs [28], and graphene [29]. The high conductivity improved by pre-treatment enabled $\mathrm{AgNWs}$ to become excellent transparent electrode materials especially at high transmittance.

The washed AgNWs not only improved the conductivity of the film electrodes, but also overcome the substrate limitation induced by the post-treatment and could be directly coated on various substrates with different shapes and properties. The W90-AgNWs were coated on the glass beaker, PET bottle, tissue paper, and bacterial cellulose substrate (Fig. S6). These AgNW films on different substrates all showed high conductivity without any posttreatment. The flat PET substrate-based electrodes with W90-AgNWs were incorporated into a capacitive pressure sensor for validation in a practical application. $5 \mathrm{wt} \% \mathrm{PVP}$ solution in ethanol was coated on the AgNW electrodes and dried as the dielectric interlayer, and two pieces of sandwiched PET/AgNWs/PVP structures were stacked as shown in Fig. S7. This kind of structure could be fabricated in different shapes and sizes, on rigid or flexible substrates. First, the sensor employing two pieces of $93.7 \%$-transmittance AgNW electrodes was investigated (see Fig. S8). Pulsed pressures of 0.5 and $1.0 \mathrm{kPa}$ were used to test the response of the capacitive sensor as shown in Fig. 6a. Under intermittent pressure stimuli of $0.5 \mathrm{kPa}$, the capacitance changed rapidly from ca. 0.1 to $0.24 \mathrm{nF}$, with good reproducibility throughout the test. When the pressure was increased to $1.0 \mathrm{kPa}$, a larger response of $0.35 \mathrm{nF}$ was obtained with good stability. Under mechanical pressure, the distance between the overlapping areas of upper and lower electrodes decreased rapidly resulting in immediate
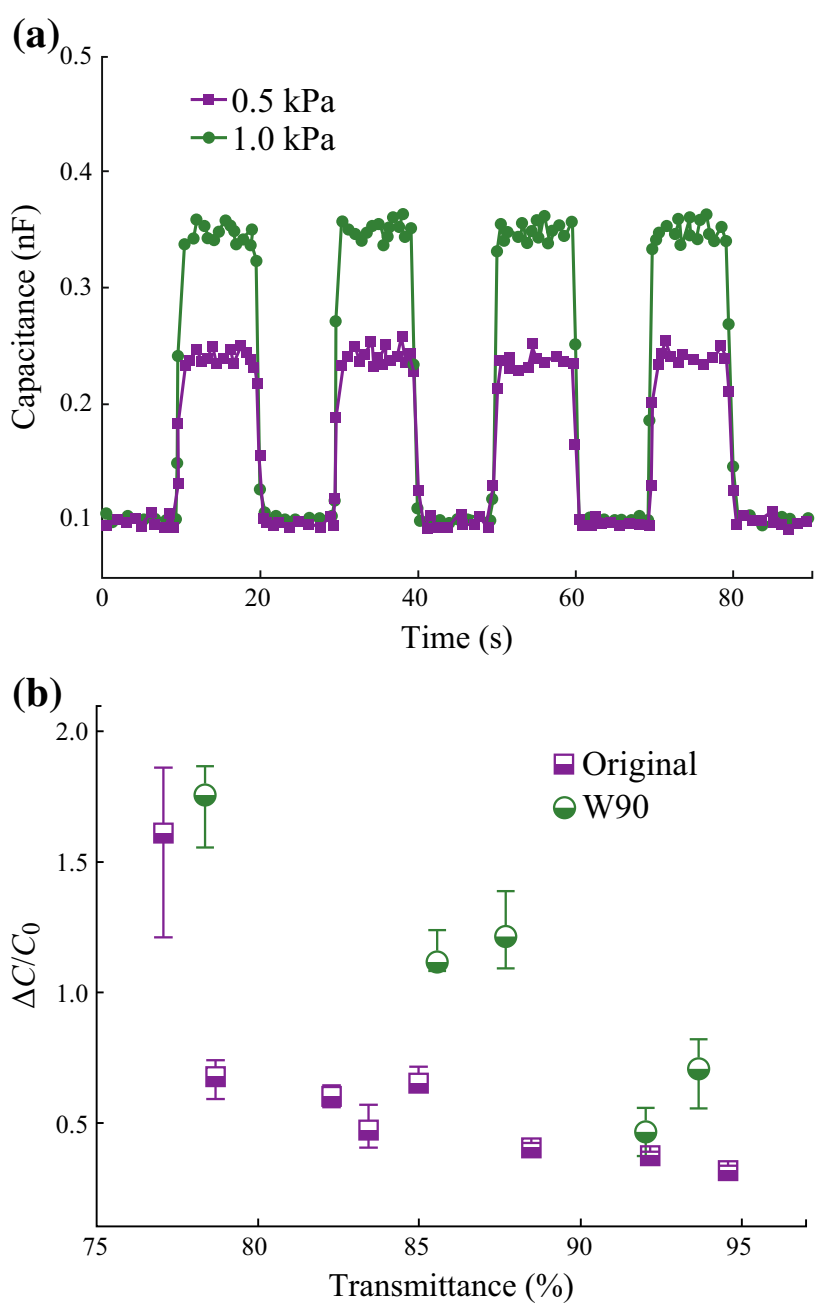

Fig. 6 Electrodes with modified AgNWs were employed in capacitive pressure sensor application. a Capacitance response to pulsed pressure of 0.5 and $1.0 \mathrm{kPa}$, respectively. b Capacitance change $\Delta C /$ $C_{0}$ of the pressure sensor with modified and unmodified AgNWs at various transmittances

changes in the capacitance [30]. Figure $6 \mathrm{~b}$ compares the change in capacitance under a pressure of $0.3 \mathrm{kPa}$ for sensors assembled using electrodes with different transmittances. The sensor using washed AgNWs showed a higher capacitance change $\Delta C / C_{0}\left(C_{0}\right.$ is defined as the original capacitance without any pressure, and $\Delta C$ is defined as the difference between the capacitance with pressure and $C_{0}$ ) at all transmittances compared to the sensor using untreated $\mathrm{AgNWs}$, suggesting that the sensor using $\mathrm{W} 90-\mathrm{AgNWs}$ is more sensitive to external pressure due to the improved contact resistance between AgNWs. The high-performance sensor was successfully achieved with the pre-treated AgNWs, and this pre-treatment method also provides an effective and simple route to enhance the performance of other devices based on transparent electrodes. 


\section{Conclusions}

The conductivity of AgNW transparent electrode has been dramatically improved at high optical transmittance by a simple and rapid washing method without any post-treatment. In this washing pre-treatment process, increasing the number of washing cycles leads to the gradual reduction in the thickness of PVP layer and the corresponding decrease in the sheet resistance especially at high transmittance. Washing temperature and solvent type are also important factors in the pre-treatment process. Therefore, AgNW electrodes with sheet resistances of 15.6 and $204 \Omega \mathrm{sq}^{-1}$ at transmittances of 90 and $97.5 \%$, respectively, were produced without any post-treatment at room temperature. A capacitive pressure sensor based on the pre-treated AgNWs that performs with high sensitivity, reproducibility, and transparency, is demonstrated. The AgNW ink after washing pre-treatment also avoids the substrate limitation induced by the usual post-treatment and greatly expands the application of AgNW electrode on various substrates.

Acknowledgments This work was partly supported by Showa Denko Co. Ltd, Grant-in-Aid for Scientific Research (Kaken S, 24226017) and COI Stream Project. J. Wang acknowledges the financial support from China Scholarship Council for his $\mathrm{PhD}$ research in Osaka University.

Open Access This article is distributed under the terms of the Creative Commons Attribution License which permits any use, distribution, and reproduction in any medium, provided the original author(s) and the source are credited.

\section{References}

1. X. Li, D. Zhang, S. Chen, H. Zhang, Z. Sun, S. Huang, X. Yin, Dye-sensitized solar cells with higher Jsc by using polyvinylidene fluoride membrane counter electrodes. Nano-Micro Lett. 3(3), 195-199 (2011). doi:10.3786/nml.v3i3.p195-199

2. Y.H. Kim, J. Lee, S. Hofmann, M.C. Gather, L. Müller-Meskamp, K. Leo, Achieving high efficiency and improved stability in ITO-free transparent organic light-emitting diodes with conductive polymer electrodes. Adv. Funct. Mater. 23(30), 3763-3769 (2013). doi:10.1002/adfm.201203449

3. J. Lee, P. Lee, H.B. Lee, S. Hong, I. Lee, J. Yeo, S.S. Lee, T.S. Kim, D. Lee, S.H. Ko, Room-temperature nanosoldering of a very long metal nanowire network by conducting-polymerassisted joining for a flexible touch-panel application. Adv. Funct. Mater. 23(34), 4171-4176 (2013). doi:10.1002/adfm. 201203802

4. W. Hu, X. Niu, R. Zhao, Q. Pei, Elastomeric transparent capacitive sensors based on an interpenetrating composite of silver nanowires and polyurethane. Appl. Phys. Lett. 102(8), 083303 (2013). doi:10.1063/1.4794143

5. D.S. Hecht, L. Hu, G. Irvin, Emerging transparent electrodes based on thin films of carbon nanotubes, graphene, and metallic nanostructures. Adv. Mater. 23(13), 1482-1513 (2011). doi:10. 1002/adma.201003188
6. Y. Zhi, G. Rungang, H. Nantao, C. Jing, C. Yingwu, Z. Liying, W. Hao, K. Eric Siu-Wai, Z. Yafei, The prospective 2D graphene nanosheets: preparation, functionalization and applications. Nano-Micro Lett. 4(1), 1-9 (2012). doi:10.3786/nml.v4i1.p1-9

7. J.G. Tait, B.J. Worfolk, S.A. Maloney, T.C. Hauger, A.L. Elias, J.M. Buriak, K.D. Harris, Spray coated high-conductivity PEDOT: PSS transparent electrodes for stretchable and mechanically-robust organic solar cells. Sol. Energy Mater. Sol. Cells 110, 98-106 (2013). doi:10.1016/j.solmat.2012.09.005

8. T. Tokuno, M. Nogi, J. Jiu, T. Sugahara, K. Suganuma, Transparent electrodes fabricated via the self-assembly of silver nanowires using a bubble template. Langmuir 28(25), 9298-9302 (2012). doi:10.1021/la300961m

9. M. Liu, Z. Wu, W.M. Lau, J. Yang, Recent advances in directed assembly of nanowires or nanotubes. Nano-Micro Lett. 4(3), 142-153 (2012). doi:10.3786/nml.v4i3.p142-153

10. L. Yang, T. Zhang, H. Zhou, S.C. Price, B.J. Wiley, W. You, Solution-processed flexible polymer solar cells with silver nanowire electrodes. ACS Appl. Mater. Interfaces 3(10), 4075-4084 (2011). doi:10.1021/am2009585

11. L. Daniel, G. Gaël, M. Céline, C. Caroline, B. Daniel, S. JeanPierre, Flexible transparent conductive materials based on silver nanowire networks: a review. Nanotechnology 24(45), 452001 (2013). doi:10.1088/0957-4484/24/45/452001

12. J. Jiu, T. Araki, J. Wang, M. Nogi, T. Sugahara, S. Nagao, H. Koga, K. Suganuma, E. Nakazawa, M. Hara, H. Uchida, K. Shinozaki, Facile synthesis of very-long silver nanowires for transparent electrodes. J. Mater. Chem. A 2(18), 6326-6330 (2014). doi:10.1039/c4ta00502c

13. J. Jiu, T. Sugahara, M. Nogi, K. Suganuma, Ag nanowires: largescale synthesis via a trace-salt-assisted solvothermal process and application in transparent electrodes. J. Nanopart. Res. 15(4), 1588 (2013). doi:10.1007/s11051-013-1588-3

14. S. De, T.M. Higgins, P.E. Lyons, E.M. Doherty, P.N. Nirmalraj, W.J. Blau, J.J. Boland, J.N. Coleman, Silver nanowire networks as flexible, transparent, conducting films: extremely high DC to optical conductivity ratios. ACS Nano 3(7), 1767-1774 (2009). doi:10.1021/nn900348c

15. J. Lee, I. Lee, T.S. Kim, J.Y. Lee, Efficient welding of silver nanowire networks without post-processing. Small 9, 2887-2894 (2013). doi:10.1002/smll.201203142

16. T. Tokuno, M. Nogi, M. Karakawa, J. Jiu, T.T. Nge, Y. Aso, K. Suganuma, Fabrication of silver nanowire transparent electrodes at room temperature. Nano Res. 4(12), 1215-1222 (2011). doi:10. 1007/s12274-011-0172-3

17. J. Jiu, T. Sugahara, M. Nogi, T. Araki, K. Suganuma, H. Uchida, K. Shinozaki, High-intensity pulse light sintering of silver nanowire transparent films on polymer substrates: the effect of the thermal properties of substrates on the performance of silver films. Nanoscale 5(23), 11820-11828 (2013). doi:10.1039/ c3nr03152g

18. P.C. Hsu, S. Wang, H. Wu, V.K. Narasimhan, D. Kong, H.R. Lee, Y. Cui, Performance enhancement of metal nanowire transparent conducting electrodes by mesoscale metal wires. Nat. Commun. 4, 2522 (2013). doi:10.1038/ncomms3522

19. L. Hu, H.S. Kim, J.-Y. Lee, P. Peumans, Y. Cui, Scalable coating and properties of transparent, flexible, silver nanowire electrodes. ACS Nano 4(5), 2955-2963 (2010). doi:10.1021/nn1005232

20. P. Lee, J. Lee, H. Lee, J. Yeo, S. Hong, K.H. Nam, D. Lee, S.S. Lee, S.H. Ko, Highly stretchable and highly conductive metal electrode by very long metal nanowire percolation network. Adv. Mater. 24(25), 3326-3332 (2012). doi:10.1002/adma.201200359

21. T. Araki, J. Jiu, M. Nogi, H. Koga, S. Nagao, T. Sugahara, K. Suganuma, Low haze transparent electrodes and highly conducting air dried films with ultra-long silver nanowires 
synthesized by one-step polyol method. Nano Res. 7(2), 236-245 (2014). doi:10.1007/s12274-013-0391-x

22. P. Peng, A. Hu, H. Huang, A.P. Gerlich, B. Zhao, Y.N. Zhou, Room-temperature pressureless bonding with silver nanowire paste: towards organic electronic and heat-sensitive functional devices packaging. J. Mater. Chem. 22(26), 12997-13001 (2012). doi:10.1039/C2JM31979A

23. Z. Zhang, B. Zhao, L. Hu, PVP protective mechanism of ultrafine silver powder synthesized by chemical reduction processes. J. Solid State Chem. 121(1), 105-110 (1996). doi:10.1006/jssc. 1996.0015

24. V. Bühler, Polyvinylpyrrolidone excipients for pharmaceuticals, vol. 2 (Springer, Berlin, 2005), pp. 5-124. doi:10.1007/3-54027090-6_2

25. S. De, J.N. Coleman, The effects of percolation in nanostructured transparent conductors. Mater. Res. Soc. Bull. 36(10), 774-781 (2011). doi:10.1557/mrs.2011.236

26. J. Lee, P. Lee, H. Lee, D. Lee, S.S. Lee, S.H. Ko, Very long Ag nanowire synthesis and its application in a highly transparent, conductive and flexible metal electrode touch panel. Nanoscale 4(20), 6408-6414 (2012). doi:10.1039/c2nr31254a
27. T.M. Barnes, M.O. Reese, J.D. Bergeson, B.A. Larsen, J.L. Blackburn, M.C. Beard, J. Bult, J. van de Lagemaat, Comparing the fundamental physics and device performance of transparent, conductive nanostructured networks with conventional transparent conducting oxides. Adv. Energy Mater. 2(3), 353-360 (2012). doi:10.1002/aenm.201100608

28. S.H. David, M.H. Amy, L. Roland, H. Liangbing, M. Bryon, C. Chad, R. Steven, High conductivity transparent carbon nanotube films deposited from superacid. Nanotechnology 22(7), 075201 (2011). doi:10.1088/0957-4484/22/7/075201

29. S. Bae, H. Kim, Y. Lee, X. Xu, J.S. Park, Y. Zheng, J. Balakrishnan, T. Lei, H. Ri Kim, Y.I. Song, Y.J. Kim, K.S. Kim, B. Ozyilmaz, J.H. Ahn, B.H. Hong, S. Iijima, Roll-to-roll production of 30-inch graphene films for transparent electrodes. Nat. Nanotechnol. 5(8), 574-578 (2010). doi:10.1038/nnano.2010.132

30. C. Mayousse, C. Celle, E. Moreau, J.F. Mainguet, A. Carella, J.P. Simonato, Improvements in purification of silver nanowires by decantation and fabrication of flexible transparent electrodes. Application to capacitive touch sensors. Nanotechnology 24(21), 215501 (2013). doi:10.1088/0957-4484/24/21/215501 